\title{
The Autonomous Photovoltaic MarXbot
}

Florian Vaussard, Philippe Rétornaz, Martin Liniger, and Francesco Mondada

\begin{abstract}
Domestic service robots are currently powered by the mains electricity. The growing multiplication of such devices negatively impacts our environment. In this study, we show the feasibility of harvesting energy from natural light in an indoor environment. The design of the harvester is carefully carried out using an experimental characterisation of several solar panels, while the boost converter is optimised to operate at low-light intensities and the robot is enhanced for lowpower operations. The resulting harvester is then thoroughly characterised. Finally, a phototaxis experiment is conducted, proving the feasibility of recharging the robot solely by using this form of energy. The possibility of embedding energy harvesting in indoor mobile robots radically changes the potential impact of this technology in our society.
\end{abstract}

\section{Introduction}

Domestic service robots are a rapidly growing market. In 2010, at least 1.45 million units have been sold, mostly vacuum cleaners [1]. This is roughly a $40 \%$ increase compared to the previous year. The total population can be estimated at around seven million units, even if this figure is subject to uncertainty.

All these devices are powered by batteries. In the best case, the robot can recharge itself using the mains electricity and a docking station. In the worst case, primary batteries must be periodically replaced. If these robots are in use only $1 \%$ of the time, given the mentioned population, this equals 600 million hours of activity per year. If we take a reasonable power consumption of $10 \mathrm{~W}$, this represents $6 \mathrm{GW} \mathrm{h}$ per year, or 510 Tonne of Oil Equivalent (TOE). Our long-term goal is to replace

Florian Vaussard · Philippe Rétornaz · Martin Liniger · Francesco Mondada EPFL - STI - LSRO, Station 9, 1015 Lausanne, Switzerland

e-mail: firstname. lastnamedepfl.ch 
most of this power with renewable energy, for example by harvesting the energy from the surrounding environment.

In a previous study, we considered several types of energy, and showed how some of them are highly promising, such as indoor solar energy or mechanical work produced by humans [14]. The indoor environment is, however, demanding. Compared to outdoors, the house is protected against environmental phenomena, leading to scarce energy resources. In the case of a robot performing periodic tasks at a low duty cycle-like cleaning, watering the plants or patrolling-renewable energies can however account for a fair part of the total energy budget. This is what we want to assess in practice.

In this study, we present the results achieved using a prototype of a photovoltaic robot for indoor use. This energy was chosen for its high potential, especially near windows and other bright places exposed to the sun. A power density of at least $300 \mathrm{~W} \mathrm{~m}^{-2}$ behind a window was assessed during good weather in September using a solarimeter $\left(46^{\circ}\right.$ of latitude). This is two orders of magnitude above the indoor lighting.

There are a number of challenges when scavenging the solar energy directly from a mobile indoor robot. In this study, we address the mechatronics integration, with the main purpose of efficiently moving and orienting the panel, so as to maximise the collected energy. Another problem is the localization of the best spots for being recharged. We show here a simple solution based on phototaxis. More complex situations will be addressed separately.

The key points of our design are first introduced. The prototyped harvester is then validated under well-known conditions. Finally, we prove the feasibility of recharging the robot, using an indoor experiment.

\section{Prior Works}

A number of outdoor robots are effectively using solar energy, the most notable ones being the three Mars rovers-Sojourner, Spirit, Opportunity—as well as the Antarctic rover "Cool Robot". The Mars rovers were fitted with expensive GaAs/Ge and triple-junction solar panels, placed horizontally on top of the robots and providing the necessary power for a few hours per day $[12,13]$. The operating point of the solar panels is approximated using environmental conditions and a priori knowledge.

"Cool Robot" is designed to operate in Antarctic conditions [7, 8]. The power budget has been carefully modelled, including the snow-reflected component, providing a good margin for long-term polar experiments [6]. Such experiments, however, have yet to be conducted.

In the case of indoor robots, there exists only a few rigorous designs. Results are often restricted and inconclusives. Testing conditions are not always well defined, leading to non-reproducible experiments. Worthy designs include the miniature legged inchworm of Hollar [5]. It was able to move forward $3 \mathrm{~mm}$ in 30 minutes under the light provided by an optical fiber. The miniature robot "Alice" was also 
fitted with solar panels, operating under a 3000 ANSI lumens beamer [2]. Power densities of 7.1 and $27.8 \mathrm{~W} \mathrm{~m}^{-2}$ were achieved using crystalline silicon and thin film amorphous silicon cells. The overall power balance is, however, unknown.

The problem of the panel's orientation is often not considered by researchers, simplified to a horizontal placement. The tracked robot of Hartono is fitted with a fixed-tilt panel and four photodiodes at each corner [4]. These inputs are fed into a neuronal network, which controls the driving motors and switches between several survival strategies. This setup has been tested for 33 hours in uncharacterised outdoor conditions.

In summary, a rigorous design, validated by by a proof a concept experiment, was not yet demonstrated in the literature. We propose to fill in the gap in this study, using a simple approach to locate the energy source.

\section{Design}

Our design was driven by several key points. First, the system should minimise the consumed energy, while being able to move in an indoor environment. It should also maximise the illumination on the solar panel and efficiently convert the incoming power into usable energy.

We are using the small marXbot robot as the mobile platform [3]. It is a highlymodular, low-power mobile robot. Subsystems can be put into sleep when unused, and we are thus able to minimise the wasted energy when recharging. Its rich set of sensors provides the robot with advanced navigation capabilities, like a low-cost range scanner and an embedded ARM-based computer [9] (Fig. 1a). For our experiments, we will solely use the mobile base, as pictured in Fig. $1 \mathrm{~b}$.

The characterisation of solar panels is performed by the manufacturers under the Air Mass (AM) 1.5 model, with a standard illumination of $1000 \mathrm{~W} \mathrm{~m}^{-2}$ at sea level. Our conditions are far from this norm: low light intensity and a spectrum filtered by a window can heavily influence the efficiency, as shown by [11]. For our design, we have compared four photovoltaic panels, two made of monocrystalline silicon and two made of thin film amorphous silicon.

During the characterisation process, the I/V curve was recorded using a variable resistive load. We considered two light conditions: the sunlight, as received behind a south-oriented window, and an artificial light. The monocrystalline cell AH508480G (Sunways) outperformed the others. Table 1 shows the efficiency of this cell under the two test conditions.

This single cell is providing a high current (8 A under AM 1.5) at low voltage $\left(\mathrm{V}_{O C}=0.63 \mathrm{~V}\right)$. Due to space limitations, we were unable to mount several ones in a serial configuration. A boost DC/DC converter has been specially optimised for low-light conditions, based on a model of the prevalent losses at the operating point $\left(150 \mathrm{~W} \mathrm{~m}^{-2}\right)$. As shown in Sec. 4 , the measured efficiency is above $85 \%$ at low light intensity. 


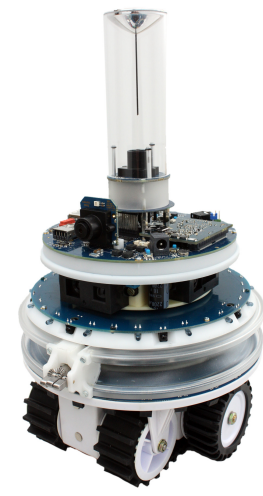

(a)

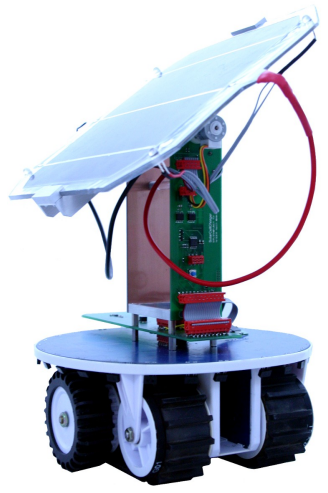

(b)

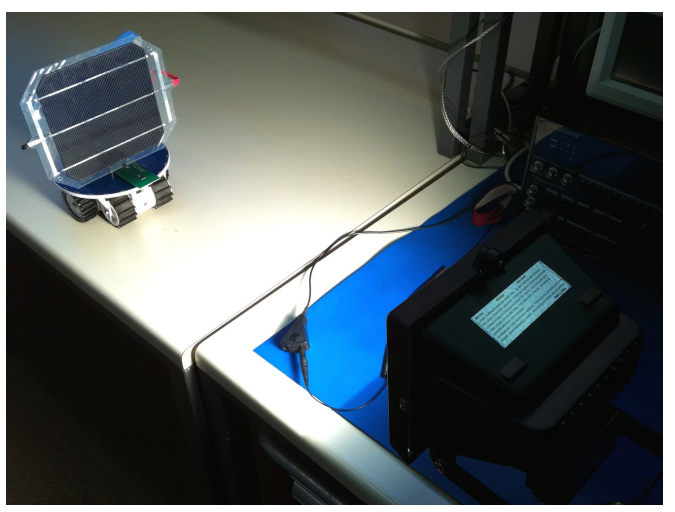

(c)

Fig. 1: The complete marXbot robot (a) and its stripped-down photovoltaic version (b). The photovoltaic marXbot during the efficiency test (c).

Table 1: Experimental cell characterisation

\begin{tabular}{lcccc}
\hline $\begin{array}{l}\text { Light } \\
\text { Source }\end{array}$ & $\begin{array}{c}\text { Illumination } \\
\mathbf{G} \\
\left(\mathrm{W} \mathrm{m}^{-2}\right)\end{array}$ & $\begin{array}{c}\text { Cell Power } \\
\mathbf{P}_{\text {cell }} \\
(\mathrm{W})\end{array}$ & $\begin{array}{c}\text { Area } \\
\mathbf{A}_{\text {cell }} \\
\left(\mathrm{m}^{2}\right)\end{array}$ & $\begin{array}{c}\text { Efficiency } \\
\boldsymbol{\eta}_{\text {cell }}\end{array}$ \\
\hline Sun $^{a}$ & 150 & 0.36 & $2.43 \cdot 10^{-2}$ & 0.099 \\
Artificial $^{b}$ & 148 & 0.30 & $2.43 \cdot 10^{-2}$ & 0.083 \\
\hline
\end{tabular}

${ }^{a}$ Behind a window ${ }^{b}$ OSRAM $60 \mathrm{~W}$ incandescent "daylight" bulb 
To control the photovoltaic subsystem, we are using a two-layer architecture. A low-power 8 bits microcontroller is controlling the DC/DC converter to perform the Maximum Power Point Tracking (MPPT). A 16 bits microcontroller is in charge of the high-level control during the experiment, able to put itself and the robot into deep sleep when appropriate. By using the event-based framework Aseba [10], we can easily prototype behaviours and log data.

The alignment of the cell with the light source is crucial to maximise the collected power. While most designs neglect this point, we addressed this issue by adding a rotational Degree of Freedom (DOF) to the panel, leading to a total of five DOFs, when taking into account the mobility of the system. Several mechanical solutions have been evaluated, based on the energy required to orient the panel. The efficiency of the mechanical transmission appears to be the limiting factor. The final system is shown in Fig. 1b. The rotation axis is placed closed to the centre of mass, in order to minimise the gravitational couple on the motor. A stepper motor is in direct drive with the axis.

Four photodiodes were placed at each corner, to allow the localization of the light source using a gradient ascent strategy.

\section{Results}

An experimental power model of the marXbot was introduced in [3]. For our stripped-down version, the idle consumption is $0.9 \mathrm{~W}$ with modules enabled. The total power consumed when moving can be expressed as a quadratic function of the speed. The electronics have been further improved during this study to enable a deep sleep state. The power supply of each module can be actively turned off, decreasing the total power consumption to $40 \mathrm{~mW}$.

\subsection{Harvester Characterisation}

A simple model of the harvester is shown in Fig. 2. The system was characterised using a halogen light of $60 \mathrm{~W}$ at a variable distance (Fig. 1c). The illumination is measured with a solarimeter at the centre of the cell. The power produced by the cell $\mathrm{P}_{\text {cell }}$, as well as the power at the output of the converter $\mathrm{P}_{\mathrm{DC} / \mathrm{DC}}$, are plotted against the illumination $\mathrm{G}$ in Fig. 3a.

$P_{\text {cell }}$ appears to be linear $\left(R^{2}=0.9996\right)$, as shown by the linear regression

$$
P_{\text {cell }}=a_{\text {cell }} G+b_{\text {cell }}=3.02 \cdot 10^{-3} G+7.4 \cdot 10^{-3}(\mathrm{~W}) .
$$

This implies a constant cell efficiency on the studied range, which equals

$$
\eta_{\text {cell }}=a_{\text {cell }} / A_{\text {cell }}=0.124
$$




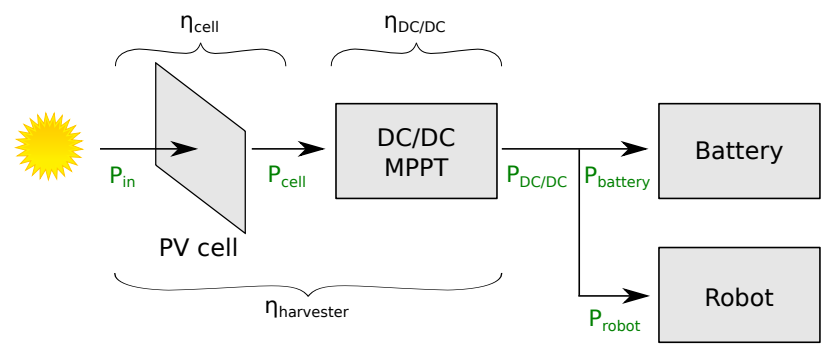

Fig. 2: Simple model of the harvester.

On the other side, $\mathrm{P}_{\mathrm{DC} / \mathrm{DC}}$ depends on the efficiency of the DC/DC converter

$$
P_{\mathrm{DC} / \mathrm{DC}}=\eta_{\mathrm{DC} / \mathrm{DC}} P_{\text {cell }}=\eta_{\text {harvester }} P_{\mathrm{in}} .
$$

Efficiencies $\eta_{\mathrm{DC} / \mathrm{DC}}$ and $\eta_{\text {harvester }}$ are plotted in Fig. $3 \mathrm{~b}$ and 3c, respectively.

The achieved efficiency is especially good at low illumination. The harvester's efficiency is above $10 \%$ in the range from 30 to $110 \mathrm{~W} \mathrm{~m}^{-2}$. At higher illuminations, the high current delivered by the cell increases the Joule losses inside the self and the high-side transistor of the DC/DC converter.

\subsection{Phototaxis Experiment}

In order to show the viability of our design, a phototaxis experiment was conducted using the setup of Fig. 4a. A halogen lamp is placed in one corner of the arena, with a fixed tilt angle. The distribution of the light's intensity, as measured by the solarimeter, is shown in Fig. $4 \mathrm{~b}$. There is a peak, with a value of $161 \mathrm{~W} \mathrm{~m}^{-2}$. According to the efficiency of our harvester, we should be able to extract $0.37 \mathrm{~W}$ under optimal placement.

A simple P controller was implemented using the photodiodes to control the heading of the robot, while moving autonomously towards the source. When the derivative of the cell's power flattens out, the robot stops its progress and orients the panel towards the light.

The results are shown in Fig. $4 \mathrm{c}$ and $4 d$, using two different time scales. At small scale, the power used to move forward is around $2 \mathrm{~W}$, whereas it uses up to $5 \mathrm{~W}$ when turning on spot. This is due to the poor efficiency of the tracked robot when turning on a carpet. The power delivered by the cell increases as the robot is reaching its goal. The power delivered by the DC/DC converter reaches a maximum of $0.297 \mathrm{~W}$ after $44 \mathrm{~s}$. This is $80 \%$ of the theoretical maximum.

At a larger time scale, the embedded charge counter first begins to decrease, due to the energy required to move, reaching its minimum at $45 \mathrm{~s} ; 100 \mathrm{~J}$ have been used for this part. Then, the battery starts charging. The energy equilibrium is reached after ten minutes. Thereafter, the battery starts gaining energy compared to its initial 


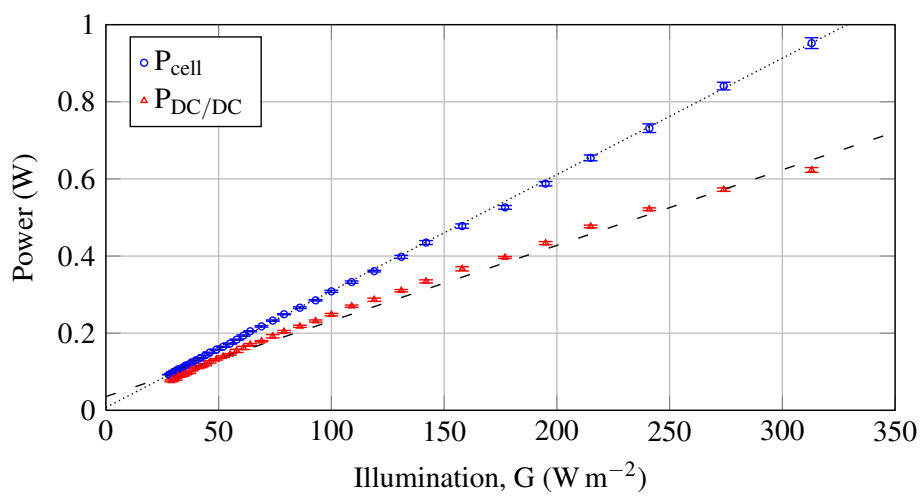

(a)

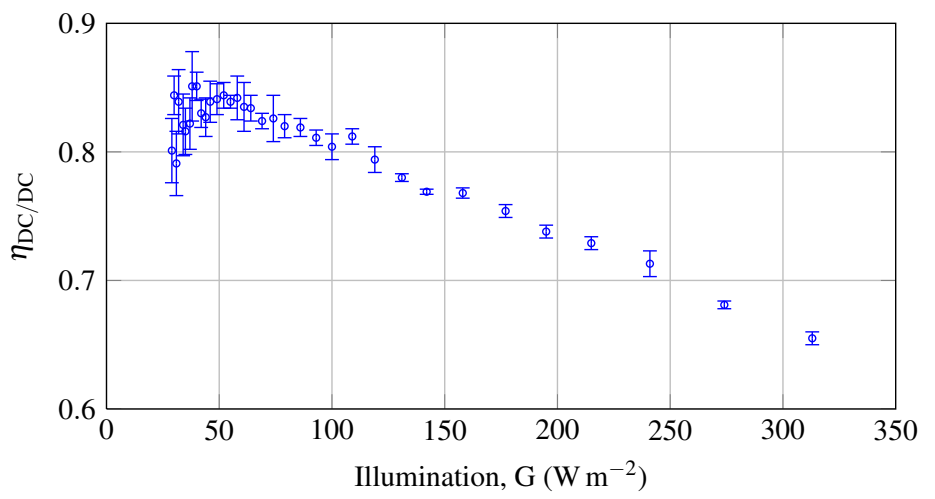

(b)

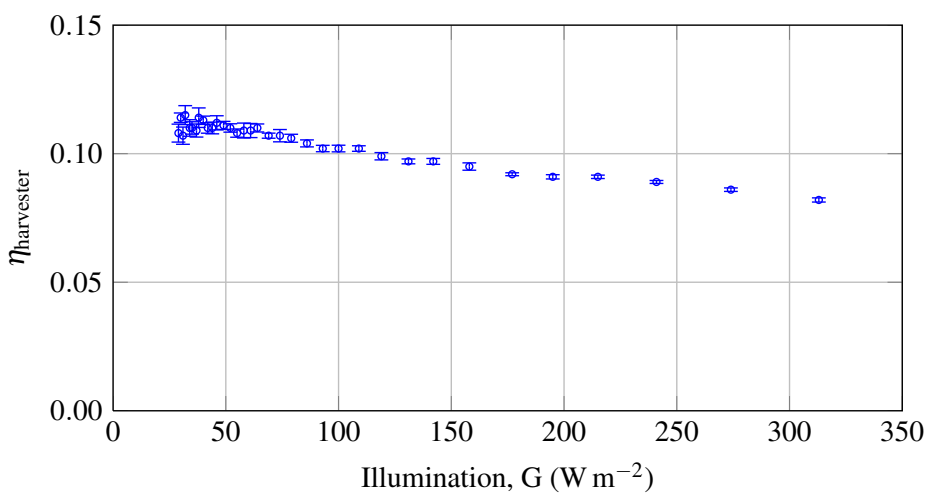

(c)

Fig. 3: Experimental results of the solar harvester under halogen illumination. 


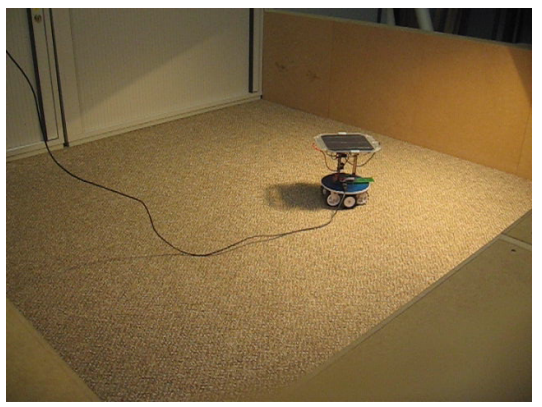

(a)

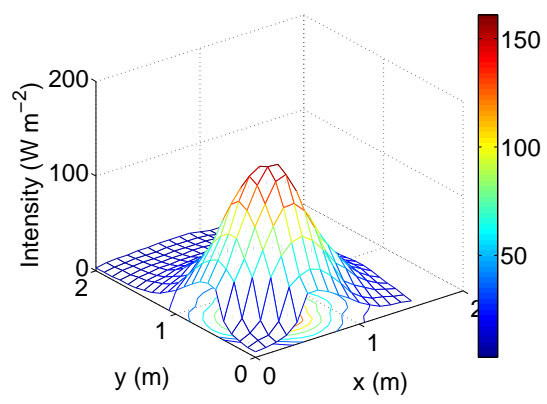

(b)

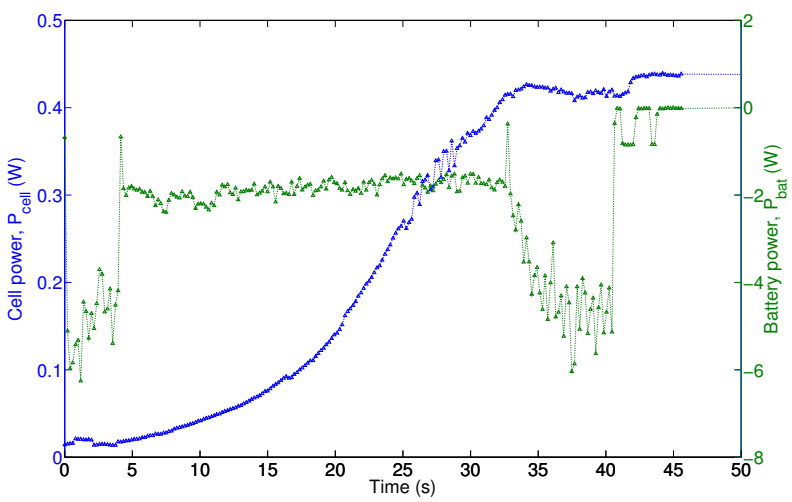

(c)

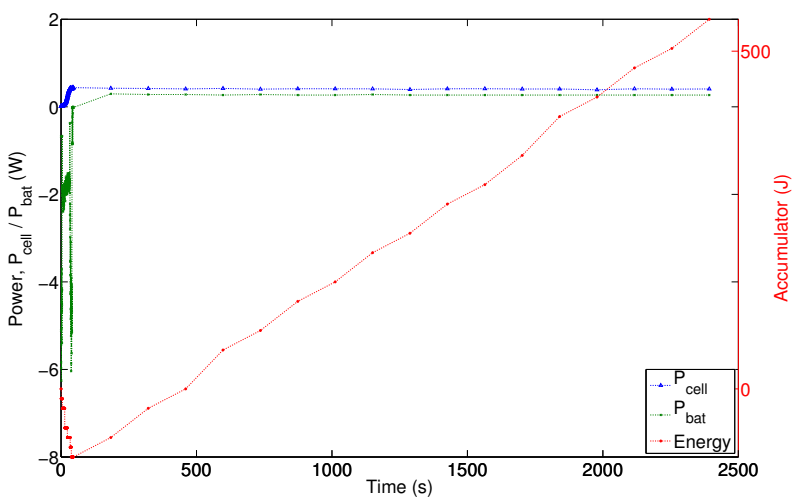

(d)

Fig. 4: Phototaxis experiment. (a) Experimental setup. (b) Light intensity distribution. (c) Energy harvesting: maximisation phase. (d) Energy harvesting: recharge phase. 
state. At the end, after 40 minutes of experiment, we have a net gain of $547 \mathrm{~J}$. A total of 130 hours would be needed to recharge the robot, given a battery of $40 \mathrm{Wh}$, for an autonomy of eight hours when moving. Thus, for one hour of autonomy, we need 16 hours of recharge, about one week under reasonable assumptions. This matches our design target of a domestic robot operating a few hours per week. It is close to the theoretical conclusions we draw in [14].

\section{Conclusion}

We have shown the possibility of autonomously recharging our robot using an indoor experiment and a phototaxis strategy. The chosen light's intensity is what we are expecting from the sun entering the house through windows.

The next step is to test our design in a less synthetic setup, using the real sunlight. In this case, a simple phototaxis strategy might not be enough, and we are planning to integrate the energy-mapping problem inside the Simultaneous Localization And Mapping (SLAM) framework.

As pointed out, a tracked robot is rather energy inefficient when moving, especially when turning on spot. An adequate platform is also very important, considering the targeted environment. An efficient locomotion principle has to be adopted when moving indoor. Simple wheels are not a good solution, as they usually get stuck in carpets and other similar obstacles. This will also be a focus of our future researchers.

Acknowledgements This research was supported by the Swiss National Science Foundation through the National Centre of Competence in Research Robotics.

\section{References}

1. International Federation of Robotics: Service Robot Statistics, http://www.ifr.org/ service-robots/statistics

2. Boletis, A., Driesen, W., Breguet, J.M., Brunete, A.: Solar cell powering with integrated global positioning system for $\mathrm{mm} 3$ size robots. In: IROS. pp. 5528-5533. IEEE (2006)

3. Bonani, M., Longchamp, V., Magnenat, S., Rétornaz, P., Burnier, D., Roulet, G., Vaussard, F., Bleuler, H., Mondada, F.: The marXbot, a miniature mobile robot opening new perspectives for the collective-robotic research. In: IROS. p. 4187-4193. IEEE (2010)

4. Hartono, P., Tabe, K., Suzuki, K., Hashimoto, S.: Strategy acquirement by survival robots in outdoor environment. In: ICRA. pp. 3571-3575. IEEE (2003)

5. Hollar, S.: A solar-powered, milligram prototype robot from a three-chip process. Ph.D. thesis, University Of California (2003)

6. Lever, J., Ray, L.: Revised solar-power budget for $\mathrm{i}_{i}$ cool robot $\mathrm{i}_{\mathrm{i}} \mathrm{i}_{i}$ polar science campaigns. Cold Regions Science and Technology 52(2), 177-190 (2008)

7. Lever, J., Ray, L., Streeter, A., Price, A.: Solar power for an antarctic rover. Hydrological processes 20(4), 629-644 (2006) 
8. Lever, J., Streeter, A., Ray, L.: Performance of a solar-powered robot for polar instrument networks. In: Robotics and Automation, 2006. ICRA 2006. Proceedings 2006 IEEE International Conference on. pp. 4252-4257. IEEE (2006)

9. Magnenat, S., Longchamp, V., Bonani, M., Rétornaz, P., Germano, P., Bleuler, H., Mondada, F.: Affordable slam through the co-design of hardware and methodology. In: Robotics and Automation (ICRA), 2010 IEEE International Conference on. p. 5395-5401. IEEE (2010)

10. Magnenat, S., Rétornaz, P., Bonani, M., Longchamp, V., Mondada, F.: ASEBA: a modular architecture for event-based control of complex robots. Mechatronics, IEEE/ASME Transactions on (99), 1-9 (2009)

11. Randall, J., Jacot, J.: Is AM1. 5 applicable in practice? Modelling eight photovoltaic materials with respect to light intensity and two spectra. Renewable Energy 28(12), 1851-1864 (2003)

12. Shirley, D., Matijevic, J.: Mars pathfinder microrover. Autonomous Robots 2(4), 283-289 (1995)

13. Stella, P., Ewell, R., Hoskin, J.: Design and performance of the mer (mars exploration rovers) solar arrays. In: Photovoltaic Specialists Conference, 2005. Conference Record of the Thirtyfirst IEEE. pp. 626-630. IEEE (2005)

14. Vaussard, F., Bonani, M., Rétornaz, P., Martinoli, A., Mondada, F.: Towards autonomous energy-wise RObjects. Towards Autonomous Robotic Systems pp. 311-322 (2011) 